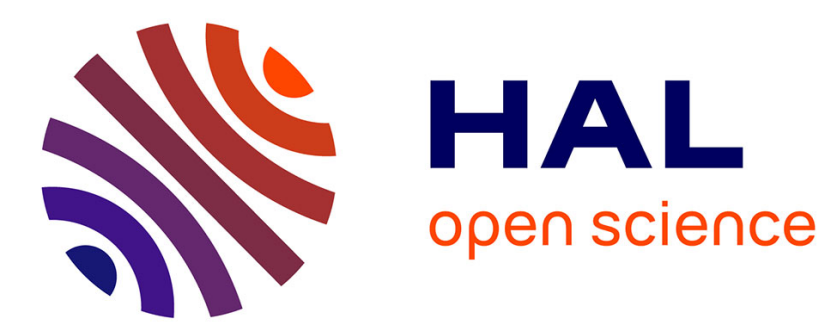

\title{
Periodic external input tunes the stability of delayed nonlinear systems: from the slaving principle to center manifolds
}

Axel Hutt, Jérémie Lefebvre

\section{To cite this version:}

Axel Hutt, Jérémie Lefebvre. Periodic external input tunes the stability of delayed nonlinear systems: from the slaving principle to center manifolds. 2013. hal-00912418

\author{
HAL Id: hal-00912418 \\ https://hal.inria.fr/hal-00912418
}

Preprint submitted on 2 Dec 2013

HAL is a multi-disciplinary open access archive for the deposit and dissemination of scientific research documents, whether they are published or not. The documents may come from teaching and research institutions in France or abroad, or from public or private research centers.
L'archive ouverte pluridisciplinaire HAL, est destinée au dépôt et à la diffusion de documents scientifiques de niveau recherche, publiés ou non, émanant des établissements d'enseignement et de recherche français ou étrangers, des laboratoires publics ou privés. 


\title{
Periodic external input tunes the stability of delayed nonlinear systems: from the slaving principle to center manifolds
}

\author{
Axel Hutt ${ }^{1}$ and Jérémie Lefebvre ${ }^{2}$ \\ 1 INRIA CR Nancy - Grand Est, 615 Rue du Jardin Botanique, 54602 \\ Villers-les-Nancy, France \\ 2 Département des Neurosciences Fondamentales, Université de Genève, CMU, 1 rue \\ Michel Servet, 1211 Genève 4, Switzerland
}

\begin{abstract}
The work illustrates a recent analysis technique that demonstrates that external periodic input affects the stability of the timeaveraged nonlinear dynamics of a delayed system. At first, the article introduces the fundamental elements of delayed differential equations and then applies these to a nonlinear delayed problem close to a transcritical bifurcation. We observe a shift of stability in the system induced by the fast periodic driving.
\end{abstract}

Keywords: slaving principle, center manifold theorem, delay equations, bifurcation theory

\section{Introduction}

Self-interactions are one of the fundamental components of complex systems. The consideration of these reentrant contributions, oftentimes exhibiting some form of latency or delay, play an important role in numerous areas of research (lasers, machining, chemistry, control), especially in models of biological systems. As such, over the last decades, retarded dynamical systems have been used to successfully describe physiological systems like the eye light pupil reflex [1], blood circulation [2] or postural motor control [3]. The influence of time lags is also ubiquitous in neuroscience, where various feedback loops have been exposed throughout neural circuitry. It has indeed been shown that delays play many important functional roles in neural systems and constitute one of the main mechanism underlying network synchronization and spatio-temporal activity patterns [4-7]. Over the past years, the question as to how spatio-temporal forcing interacts with retarded dynamics has received a vivid interest. The exact function of delays in the integration of temporally fluctuating temporal signals is still unknown, mainly because of the lack of tools the dynamical systems theory provides for the study of the stability of non-homogeneous and/or nonautonomous problems.

The center manifold theorem and the slaving principle have proven to be powerful tools in this task, and since then they have been successfully applied to 
many non-driven delayed problems [8-13]. The slaving principle may be seen as the physical equivalent, i.e. the representation in nature, of the major statement of the center manifold theorem. In this context, the center manifold theorem represents a promising candidate in the approach of the non-autonomous cases. However, the question whether the center manifold theorem may be extended to non-autonomous, i.e. forced, delayed problems is currently left poorly addressed. They are indeed indications that center manifolds do exist in infinite dimensional non-autonomous dynamical systems [14]. Furthermore, stochastic center manifold theory has been established in non-delayed noisy systems [15-17], and a similar approach has been used in the numerical analysis of non-linear ODEs, subject of time-dependent forcing, providing accurate results. However, as the need to analyse forced system arises, it is still unfortunately unclear how to apply and compute center manifolds for non-autonomous delayed feedback systems and to expose the underlying mechanism by which the unstable modes govern the dynamics. A solution to this problem would greatly enhance the possibilities of theoretical analysis of delayed systems, of prime importance in mathematical neuroscience and related fields.

In the following, we present a method that allows to compute center manifolds of delayed system with time-dependent driving. We first outline the fundamental elements of delay-differential equations and the corresponding center manifold reduction in delayed systems, summarizing the detailed discussions of $[10,18-21]$. We then present results for a periodically driven delayed model with quadratic non-linearity, and show that it describes accuratly the dynamics near a transcritical instability.

\section{General treatment of autonomous DDEs}

Consider the general autonomous scalar delay differential equation,

$$
\dot{x}(t)=f(x(t), x(t-\tau))=L(\{x(t), x(t-\tau)\})+F(\{x(t), x(t-\tau)\}),
$$

where $L$ is a linear function with $\mathrm{L}(0)=0$, and where $F$ is a non linear and sufficiently smooth function, satisfying $F(0)=D F(0)=0$. Both linear and nonlinear parts of this system may contain delayed components. In the following, we will consider the control parameter $\varepsilon$ and investigate the stability of Eq.(1) unfolded around the point $\varepsilon=0$. It will prove convenient to work with the augmented system

$$
\begin{array}{rlrl}
\dot{x}(t) & =f(x(t), x(t-\tau), \varepsilon)=L(x(t), x(t-\tau), \varepsilon)+F(x(t), x(t-\tau) \varepsilon) . \\
\frac{d \varepsilon}{d t} & = & 0 .
\end{array}
$$

This allows to take immediately into account the role played by the parameters in subsequent derivations. 


\subsection{The embedding}

In order to consider solutions $x(t)$ of Eq. (1) for $t \geq 0$, one needs a complete description of initial value problem, corresponding to the retarded dynamics into the interval $[-\tau, 0]$. This criterion implies that the map from the interval $[-\tau, 0]$ into $\mathbb{R}$ is not injective. Consequently, the system of Eq. (1) has ill-defined initial conditions, and uniqueness of solutions is not guaranteed [10]. It is therefore imperative to consider Eq. (1) in an appropriate phase space which would ensure its self-consistency. To take into account the continuous dependence of the flow $x(t)$ on the retarded dynamics, we introduce the parameter $\theta$ with $-\tau \leq \theta \leq 0$ and the new variable $z_{t}(\theta) \in \Re^{2}$, so that

$$
z_{t}(\theta) \equiv(x(t+\theta), \varepsilon)^{T} .
$$

Based on this definition, an appropriate phase space can be shown to be the Banach space of continuous maps $\mathcal{C} \equiv C([-\tau, 0], \mathbb{R} \times \mathbb{R})[10,21]$. Reformulating Eq.(1) to take into account the continuous dependence of the flow on $\theta$, we formally obtain

$$
\left[\frac{d x(t+\theta)}{d t}, \frac{d \varepsilon}{d t}\right]^{T}=\frac{d}{d t} z_{t}(\theta) \equiv \lim _{\delta t \rightarrow 0} \frac{\left(z_{t}(\theta+\delta t)\right)-z_{t}(\theta)}{\delta t},
$$

where we will distinguish instantaneous and retarded dynamics. For $-\tau \leq \theta<0$, expanding around $\delta t=0$ to first order yields [10]

$$
\frac{d}{d t} z_{t}(\theta)=\lim _{\delta t \rightarrow 0} \frac{z_{t}(\theta)+\frac{\partial z_{t}(\theta)}{\partial(\theta)} \delta t-z_{t}(\theta)}{\delta t}=\frac{\partial z_{t}(\theta)}{\partial \theta} .
$$

On the other hand, if $\theta=0$, we simply get

$$
\frac{d}{d t} z_{t}(0)=\left(\frac{d x(t)}{d t}, \frac{d \varepsilon}{d t}\right)^{T}=(f(x(t), x(t-\tau)), 0)^{T}=\left(L\left[z_{t}\right]+F\left[z_{t}\right], 0\right)^{T} .
$$

It is important to note that $L\left[z_{t}\right]$ and $F\left[z_{t}\right]$ are functionals. For instance, $L\left[z_{t}\right]$ may be written as [10]

$$
L\left[z_{t}\right]=\int_{-\tau}^{0} z_{t}(\theta) \omega(\theta) z_{t}(\theta) d \theta=\int_{-\tau}^{0} d \eta[\theta] z_{t}(\theta),
$$

with the density function $\omega(\theta)$. For the scalar DDE and a single parameter, $\omega(\theta)$ is a $2 x 2$-matrix.

We may conveniently summarize these specific cases by writing the system of Eq. (1) in $\mathcal{C}$ as a infinite dimensional ODE,

$$
\frac{d}{d t} z_{t}(\theta)=\mathcal{A}\left(z_{t}(\theta)\right)+X_{o} F\left[z_{t}(\theta)\right]
$$

where the infinitesimal generator $\mathcal{A}$ is defined by [10]

$$
\mathcal{A}\left(z_{t}(\theta)\right) \equiv \frac{\partial z_{t}(\theta)}{\partial \theta}+X_{o}\left(L\left[z_{t}\right]-\left.\frac{\partial z_{t}(\theta)}{\partial \theta}\right|_{\theta=0}\right] .
$$


The connection function $X_{o}(\theta)$ allows the simultaneous treatment of the cases $\tau \leq \theta<0$ and $\theta=0$. It is defined $a d$ hoc by

$$
X_{o}(\theta)= \begin{cases}0 & -\tau \leq \theta<0 \\ \mathbb{I} & \theta=0\end{cases}
$$

Hence, the dynamics of $(x(t+\theta), \varepsilon)^{T}=z_{t}(\theta)$ is governed by the infinite dimensional ODE in Eq. (8) appropriately defined in $\mathcal{C}$ and parameterized by $\theta$.

\subsection{Spectral analysis}

Let us investigate the spectrum $\sigma(\mathcal{A})$ of the linear operator $\mathcal{A}$ taken from Eq.(9). To this end, we consider the linearized problem of Eq. (8)

$$
\frac{d}{d t} z_{t}(\theta)=\mathcal{A}\left(z_{t}(\theta)\right)
$$

Substituting the ansatz $z_{t}(\theta)=\phi(\theta) e^{\lambda t}$ at $\theta=0$ yields the eigenvalue problem,

$$
L\left[z_{t}\right]-\lambda z_{t}(0)=\left(L\left[z_{t}\right]-\lambda \mathbb{I}\right) \phi(0) \equiv \Delta(\lambda) \phi(0)=0 .
$$

Here $\phi(\theta)$ is the eigenvector associated with the Lyapunov exponent $\lambda$. The spectrum of $\mathcal{A}$ is defined by $\sigma(\mathcal{A})=\{\lambda \in \mathbb{C} \mid \Delta(\lambda)=0\}$. For $-\tau \leq \theta<0$, we obtain an ODE on $\theta$ that defines the eigenvectors

$$
\lambda \phi(\theta) e^{\lambda t}=\phi^{\prime}(\theta) e^{\lambda t}
$$

where $\phi^{\prime}(\theta)$ denotes the derivative of $\phi$ with respect to $\theta$.

A solution of Eq.(12) is $\phi(\theta)=\phi(0) e^{\lambda \theta}$. Hence, for all the eigenvalues $\lambda_{i} \in \sigma(\mathcal{A})$, one finds an associated eigenvector $\phi_{i}(\theta)$, which constitutes a basis $\Phi(\theta)=$ $\left[\phi_{1}(\theta), \phi_{2}(\theta), \ldots\right]$. This basis spans $\mathcal{C}$, and as such, we can choose to write any state vector $v \in \mathcal{C}$ in terms of the eigenbasis of $\mathcal{A}$.

However, it is possible that the basis $\Phi$ is neither orthogonal or normalized. Thus, consider the adjoint basis $\Psi^{\dagger}(s)=\left[\psi_{1}^{\dagger}(s), \psi_{2}^{\dagger}(s), \ldots\right]$ where $\psi_{i}^{\dagger}(s)$ are eigenvectors of the adjoint linear problem

$$
\left.\frac{d}{d t} y_{t}(s)=\mathcal{A}^{\dagger}\left(y_{t}\right)\right)=-A\left(y_{t}\right)= \begin{cases}-\frac{\partial y_{t}(s)}{\partial s} & 0>s \geq \tau \\ -L\left[y_{t}\right] & s=0 .\end{cases}
$$

Note that the adjoint problem of Eq.(10) is found to be the foward problem, with $t \rightarrow-t[10,18]$. To ensure the bi-orthonormality of the eigenbases of $\mathcal{A}$ and $\mathcal{A}^{\dagger}$, we further normalize the adjoint basis by

$$
\Psi(\theta)=\left(\Psi^{\dagger}(\theta), \Phi(\theta)\right)^{-1} \Psi^{\dagger}(\theta),
$$


where we introduce the bilinear form operator $($,$) in \mathcal{C}$, defined by

$$
(a(\theta), b(\theta)) \equiv a(0) b(0)-\int_{0}^{\theta} d \theta^{\prime} \int_{-\xi}^{0} d \xi\left[d \eta\left(\theta^{\prime}\right)\right] a\left(\xi-\theta^{\prime}\right) b(\xi),
$$

with the measure $d \eta^{\prime}(\theta)$ defined in Eq. (7). This bi-linear form is playing the role of the dot product in the space of functions. Normalizing the eigenbases provides

$$
(\Psi, \Phi)(\theta)=\mathbb{I} .
$$

Considering the previous results, an arbitrary state vector $v=v(\theta)$ can be expressed in terms of the eigenbasis of (10) as

$$
v(\theta)=\Phi(\theta)(\Psi(\theta), v(\theta)) .
$$

\subsection{Phase space decomposition and subspace dynamics}

A well chosen decomposition of the spectrum can play a fundamental role characterizing instabilities of Eq. (8). Indeed, in the vicinity of an instability, we might assume, without loss of generality, that a finite number of Lyapunov exponents cross the imaginary axis while changing a control parameter and all other exponents are bounded to the left-hand plane. These bifurcating, or unstable exponents near the transition point introduce a very slow time scale, while the stable components relax much faster to their steady state. As a consequence, after a sufficiently long time, the dynamics of the system is essentially determined by the slow unstable modes: this is the essence of the slaving principle [12]. Indeed, one can choose $\sigma_{\mathcal{U}}(\mathcal{A}) \equiv\{\lambda \in \mathbb{C} \mid \operatorname{Re}(\lambda)=0\}$ which leads to $\mathcal{C}=\mathcal{U}+\mathcal{S}$, where $\mathcal{S}=\overline{\mathcal{U}}$. The space $\mathcal{U}$ is the eigenspace spanned by the eigenvectors associated with unstable Lyapunov exponents. These eigenvectors constitute a basis of $\mathcal{U}$, namely $\Phi_{\mathcal{U}}(\theta) \subset \Phi(\theta)$. This implies that there exists a complementary subspace $\mathcal{S}$, spanned by $\Phi_{\mathcal{S}}$, associated with stable Lyapunov exponents i.e. $\sigma_{\mathcal{S}}(A) \equiv\{\lambda \in \mathbb{C} \mid \operatorname{Re}(\lambda)<0\}$. Here, we label $\mathcal{U}$ the subspace spanned by the unstable eigenmodes i.e. for which the eigenvalue have a zero real part, which is analogous to the center subspace, but only the terminology differ. Following the discussion in the previous section, one can project the state vector $z_{t}(\theta)$ governed by (8) with respect to the unstable basis $\Phi_{\mathcal{U}}(\theta)$,

$$
U_{t}(\theta)=\Phi_{\mathcal{U}}(\theta)\left(\Psi_{\mathcal{U}}(\theta), z_{t}(\theta)\right)=\Phi_{\mathcal{U}}(\theta)(u(t), \varepsilon)^{T},
$$

where $(u(t), \varepsilon)^{T}=\left(\Psi_{\mathcal{U}}(\theta), z_{t}(\theta)\right)$ is a vector containing the expansion amplitudes of $z_{t}(\theta)$ with respect to the unstable eigenbasis $\Phi_{\mathcal{U}}(\theta)$. According to this phase space decomposition, the state vector $z_{t}(\theta)$ may be separated into two disjoint elements, its stable and unstable components in $\mathcal{S}$ and $\mathcal{U}$, respectively. Consequently we can write $z_{t}(\theta)=U_{t}(\theta)+s_{t}(\theta)$, where

$$
s_{t}(\theta)=z_{t}(\theta)-\Phi_{\mathcal{U}}(\theta)\left(\Psi_{\mathcal{U}}(\theta), z_{t}(\theta)\right) \equiv \Phi_{\mathcal{S}}(\theta)\left(\Psi_{\mathcal{S}}(\theta), z_{t}(\theta)\right) .
$$


Following this separation, we can project the dynamics of Eq.(3) in $\mathcal{S}$ and $\mathcal{U}$, by applying the projector (16) to Eq.(8), to obtain

$$
\Phi_{\mathcal{U}}(\theta)\left(\Psi_{\mathcal{U}}(\theta), \frac{d}{d t} z_{t}(\theta)\right)=\Phi_{\mathcal{U}}(\theta)\left(\Psi_{\mathcal{U}}(\theta), \mathcal{A}\left(z_{t}(\theta)\right)\right)+\Phi_{\mathcal{U}}(\theta)\left(\Psi_{\mathcal{U}}(\theta), X_{o} F\left[z_{t}\right]\right)
$$

Using the linearity of the bilinear form Eq.(15), the left hand side is just

$$
\begin{aligned}
\Phi_{\mathcal{U}}(\theta)\left(\Psi_{\mathcal{U}}(\theta), \frac{d}{d t} z_{t}(\theta)\right) & =\frac{d}{d t} \Phi_{\mathcal{U}}(\theta)\left(\Psi_{\mathcal{U}}(\theta), z_{t}(\theta)\right) \\
& \equiv \frac{d}{d t} U_{t}(\theta) .
\end{aligned}
$$

The projection of the linear operator $\mathcal{A}$ is simply

$$
\Phi_{\mathcal{U}}(\theta)\left(\Psi_{\mathcal{U}}(\theta), \mathcal{A}\left(z_{t}(\theta)\right)\right)=\Phi_{\mathcal{U}}(\theta)\left(\Psi_{\mathcal{U}}(\theta), \mathcal{A}\left(U_{t}(\theta)\right)\right)+\Phi_{\mathcal{U}}(\theta)\left(\Psi_{\mathcal{U}}(\theta), \mathcal{A}\left(s_{t}(\theta)\right)\right) .
$$

Then, we find with Eq.(11),

$$
\left.\Phi_{\mathcal{U}}(\theta)\left(\Psi_{\mathcal{U}}(\theta), \mathcal{A}\left(U_{t}(\theta)\right)\right)=\Phi_{\mathcal{U}}(\theta)\left(\Psi_{\mathcal{U}}(\theta), \Lambda_{\mathcal{U}} U_{t}(\theta)\right)\right)
$$

where $\Lambda_{\mathcal{U}}$ is a diagonal matrix with entries being the elements of $\sigma_{\mathcal{U}}(A)$.

Computing the term $\Phi_{\mathcal{U}}(\theta)\left(\Psi_{\mathcal{U}}(\theta), \mathcal{A}\left(s_{t}(\theta)\right)\right)$ uses the biorthonormality of the stable and unstable eigenbases. In the same spirit as in the case of the stable mode projection in equation Eq.(16), the stable component $s_{t}(\theta) \in \mathcal{S}$ of the state vector $z_{t}(\theta)$ may be written with respect to the eigenbasis of $\mathcal{S}$,

$$
S_{t}(\theta)=\Phi_{\mathcal{S}}(\theta)\left(\Psi_{\mathcal{S}}(\theta), z_{t}(\theta)\right) .
$$

Using this fact, along with $\left(\Psi_{\mathcal{U}}, \Phi_{\mathcal{S}}\right)=0$ yields

$$
\Phi_{\mathcal{U}}(\theta)\left(\Psi_{\mathcal{U}}(\theta), \mathcal{A}\left(s_{t}(\theta)\right)\right)=0 .
$$

Thus, grouping the projection over the elements in $\mathcal{U}$ and $\mathcal{S}$ in Eq.(21) and Eq.(23) gives the projected linear component of Eq.(8)

$$
\left.\Phi_{\mathcal{U}}(\theta)\left(\Psi_{\mathcal{U}}(\theta), \mathcal{A}\left(z_{t}(\theta)\right)\right)=\Phi_{\mathcal{U}}(\theta)\left(\Psi_{\mathcal{U}}(\theta), \Lambda_{\mathcal{U}} U_{t}(\theta)\right)\right)=\Lambda_{\mathcal{U}} U_{t}(\theta) .
$$

The projection over the non-linear component $X_{o} F\left[z_{t}\right]$ of Eq.(8) is computed from Eq.(15), and reads

$$
\Phi_{\mathcal{U}}(\theta)\left(\Psi_{\mathcal{U}}(\theta), X_{o} F\left[z_{t}\right]\right)=\Phi_{\mathcal{U}}(\theta) \Psi_{\mathcal{U}}(0) F\left[z_{t}\right]
$$

Thus, combining Eq. (24) and Eq. (25) for $z_{t}(\theta)=\Phi_{\mathcal{U}}(\theta)(u(t), \varepsilon)^{T}+s_{t}(\theta)$, yields the dynamics of the unstable modes of Eq. (3)

$$
\begin{array}{ccc}
\mathcal{U} \ni \frac{d u(t)}{d t} & =\Lambda_{\mathcal{U}} u(t)+\Psi_{\mathcal{U}}(0) F\left[\Phi_{\mathcal{U}} u(t)+s_{t}(\theta)\right] \\
\mathcal{U} \ni \frac{d \varepsilon}{d t} & = & 0 .
\end{array}
$$


Using the same approach, we apply the operator $\left(\mathbb{I}-\Phi_{\mathcal{U}}(\theta)\left(\Psi_{\mathcal{U}}(\theta), \cdot\right)\right.$ to the state vector $z_{t}(\theta)$ of Eq. (8) to obtain the complementary dynamics of the stable modes in $\mathcal{S}$

$$
\mathcal{S} \ni \frac{d}{d t} s_{t}(\theta)=\mathcal{A}\left(s_{t}(\theta)\right)+\left(X_{o}-\Phi_{\mathcal{U}}(\theta) \Psi_{\mathcal{U}}(0)\right) F\left[\Phi_{\mathcal{U}} u(t)+s_{t}\right] .
$$

\subsection{The time-independent center manifold reduction}

The manipulations described above can be seen as a procedure first identifying the stable and unstable manifolds, and secondly writing down the dynamics of Eq.(1) in $\mathcal{U}$ and $\mathcal{S}$ explicitly. The key idea behind this projection is first that one can reduce the dynamics of the infinite dimensional system in Eq. (8) onto the finite dimensional center eigenspace in Eq.(26) by applying the center manifold theorem, and second to get rid of delays. It provides a useful analysis platform on which one can investigate dynamic instabilities using the standard tools of linear analysis of ODEs.

Bifurcations are characterized by unstable Lyapunov exponents (i.e. exhibiting a zero-real part), or equivalently by a non-empty unstable eigenspace. The precise point in parameter space where $\sigma_{\mathcal{U}}(\mathcal{A}) \neq \emptyset$ is called the instability threshold, and can be quantitatively described by $\max _{\varepsilon} \operatorname{Re}(\lambda)=0 \mid \lambda \in \sigma(\mathcal{A})$. When this critical point is reached, the center manifold theorem applies, and the stable modes in $\mathcal{S}$ are slaved by the dynamics of the unstable modes in $\mathcal{U}$ [12]. Then

$$
s_{t}(\theta)=h(\theta, u(t), \varepsilon),
$$

holds true. Consequently, the unstable modes are further described by the order parameter equation (OPE)

$$
\frac{d u}{d t}=\Lambda_{\mathcal{U}} u+\Psi_{\mathcal{U}}(0) F[\Phi u+h(u)]
$$

While in the vicinity of an instability and given that the functional $h(u)$ is known, this system captures the dynamics of Eq.(1) entirely. Delayed components are not present anymore, and the dimensionality of this representation is finite, making the OPE very useful in the treatment of non linear DDEs. Most importantly, it is possible to reconstruct the flow $x(t)$ of the original delayed system of Eq.(1) solely from the unstable modes by

$$
x^{r}(t)=\Phi_{\mathcal{U}}(0) u(t)+h(0, u(t), \varepsilon) .
$$

Although the center manifold theorem ensures that the functional $h(\theta, u(t), \varepsilon)$ exists, it is typically not unique and usually challenging to compute explicitly. Such a derivation is often realized using algebraic manipulation softwares, and other methods $[19,20]$. 
The reconstructed flow of $x^{r}(t)$ converges to the original flow $x(t)$ as the manifold $h(\theta, u)$ gets closer to its exact form. Since $d s / d t=D_{u} h(u)(d u(t) / d t)$ from Eq.(28), the center manifold satisfies the implicit relationship

$D_{u} h(\theta, u, \varepsilon)\left[\Lambda u+\Psi_{\mathcal{U}}(0) F[\Phi u+h(u)]=\mathcal{A}(h(u))+\left(X_{o}-\Phi_{\mathcal{U}}(\theta) \Psi_{\mathcal{U}}(0)\right) F\left[\Phi_{\mathcal{U}} u+h(u)\right]\right.$,

taking into account Eqs. (26) and (27). Here $D_{u}$ denotes the partial derivative with respect to $u$.

A typical ansatz to compute $h(\theta, u, \varepsilon)$ is a polynomial expansion in powers of $u$ and the control parameter $\varepsilon$. Then sorting the terms by orders of $\varepsilon$ and $u$ yields a set of first order linear differential equations in each of the polynomial coefficients for $-\tau \leq \theta<0$. The initial conditions of these are fixed by solving Eq.(31) for $\theta=0$. The dimensionality of the differential equations in each the coefficients is the same as the codimension of the bifurcation considered. With this ansatz, one can compute $h$ up to any desired accuracy, by computing higher orders coefficients in the expansion and, hence, make $x^{r}(t)$ as close as desired to $x(t)$. However, for most applications an expansion to low order is sufficient.

\subsection{The time-dependent center manifold reduction}

Now consider the non-autonomous DDE

$$
\dot{x}(t)=f(x(t), x(t-\tau), t)=L(\{x(t), x(t-\tau)\})+F(\{x(t), x(t-\tau)\})+I(t) .
$$

Equation (32) is a non-autonomous delay-differential equation and it is a challenging problem to find conditions for its stability. Close to a bifurcation point, the analysis of such DDEs has attracted increasing attention in the last years, e.g. considering more general [18,22], deterministic [23,24] or stochastic forces [25,26]. The approach discussed in the previous sections cannot, formally, be used since the origin is no longer a fixed point and the eigenbases definition and associated phase space decomposition are not valid anymore. In addition, by virtue of the new time scales introduced by the external input, it is more difficult to identify separate time scales which is necessary in the center manifold technique.

However, when the driving $I(t)$ is small and fast compared to the relatively slow unstable modes, one may consider the fixed point of the autonomous system of Eq. (1) for the analysis of Eq. (32). This step is reasonable since previous studies on nonlinear delayed systems have shown that such driven delayed systems are stable under certain conditions in the sense of Input-to-state Stability [27]. One can then use the spectrum and subspace eigenbases from the autonomous system to project the dynamics of the non-autonmous system of Eq. (32) and subsequently obtain the non-stationary version of Eqs. (26)-(27)

$$
\mathcal{U} \ni \frac{d u(t)}{d t}=\Lambda_{\mathcal{U}} u(t)+\Psi_{\mathcal{U}}(0) F\left(\Phi_{\mathcal{U}}(\theta) u(t)+s_{t}(\theta)\right)+\Psi_{\mathcal{U}}(0) I(t)
$$




$$
\mathcal{S} \ni \frac{d}{d t} s_{t}(\theta)=\mathcal{A} s_{t}(\theta)+\left(X_{o}-\Phi_{\mathcal{U}}(\theta) \Psi_{\mathcal{U}}(0)\right)\left[F\left(\Phi_{\mathcal{U}}(\theta) u(t)+s_{t}(\theta)\right)+I(t)\right] .
$$

This result has been demonstrated formally in the case of linear non-autonomous delayed system [18]. Hence the approximation is reasonable since the amplitude of both the stable and unstable modes in the vicinity of an instability can be taken arbitrarily small by adjusting the control parameter, making the non-linear component $F$ small enough. In other words, close to the origin, the nonlinear dynamics of the system is close to its linear dynamics.

To reduce the dimensionality of the system from infinity to few modes, we proceed with the asumption that the center manifold theorem still applies close to the instability and that the functional $h$ exhibits an explicit time dependence now. This assumption has been considered successfully in non-delayed systems for quasi-periodic input [28]. Moreover the existence of time-dependent center manifolds in non-delayed systems has been proven for stochastic inputs $I(t)$ [15]. Accordingly we choose

$$
s_{t}(\theta)=h(\theta, u(t), \varepsilon, t) .
$$

As in the case of autonomous systems, the functional $h$ has yet to be at least approximated, to be of any use in the analysis of Eq.(32). In particular, now the manifold $h(\theta, u, \varepsilon, t)$ satisfies

$$
\begin{aligned}
& D_{u} h(\theta, u, \varepsilon, t)\left[\Lambda u+\Psi_{\mathcal{U}}(0) F\left[\Phi_{\mathcal{U}} u(t)+h(u, \varepsilon, t)\right]+\Psi_{\mathcal{U}}(0) I(t)\right]+\frac{\partial h(\theta, u, \varepsilon, t)}{\partial t} \\
& =\mathcal{A}(h(u, \varepsilon, t))+\left(X_{o}-\Phi_{\mathcal{U}}(\theta) \Psi_{\mathcal{U}}(0)\right)\left(F\left[\Phi_{\mathcal{U}} u(t)+h(u, \varepsilon, t)\right]+I(t)\right) .
\end{aligned}
$$

As an ansatz, we add a time-dependent correction $h_{t}$ to the expansion used in the autonomous case, such that the time-dependence in the center manifold takes the form of a fast additive perturbation

$$
h\left(\theta, u, t, t^{\prime}\right)=h_{n}(\theta, \varepsilon, u)+h_{t}(\theta, t)+\mathcal{O}(m>n ; u, \varepsilon, t),
$$

where $\mathcal{O}(m ; u, \varepsilon, t)$ denotes terms of order of magnitude $m$ in $u, \varepsilon$ and the timedependent contribution. The ansatz of Eq. (36) assumes that the autonomous center manifold $h_{n}$ of order $n$ and the correction term $h_{t}$ have similar order of magnitude. In the following we assume $\mathcal{O}\left(h_{t}\right)=2$ and the order of $h_{n}$ may be $n=2$ or $n=3$. This ansatz implies time-dependent corrections that are small compared to the amplitude of the unstable modes. This ansatz is analogous to the one used by [28] for forced non-linear ODEs, which proved to accurately reproduce the dynamics for various types of driving.

Moreover, Eq. (36) assumes that, to second order, the center manifold has a separable form in time $t$ and modes $u$ which facilitates the resolution of the resulting ODE system for $-\tau \leq \theta<0$. Indeed, the substitution of this ansatz in Eq. (35) up to quadratic order, i.e. $n=2$ leads to the same set of differential 
equations as the autonomous problem, with the notable exception of an additional slow equation in $h_{t}(\theta, t)$ of order 2 . The terms in $h_{t}$ are decoupled from the autonomous contribution $h_{n}(\theta, u)$ and obey

$$
\frac{\partial h_{t}(\theta, t)}{\partial t}=\frac{\partial h_{t}(\theta, t)}{\partial \theta}-\Phi(\theta) \Psi(0) I(t) .
$$

Here we chose $\mathcal{O}(I(t))=2$ Equation (37) is a linear first order non-homogeneous partial differential equation of the time correction coefficient $h_{t}$, which may be solved using the method of characteristics, given that $I(t)$ and the entries of the bases $\Phi(\theta)$ and $\Psi(\theta)$ are smooth enough. To solve Eq. (37), we have to distinguish the two cases:

- for $t+\theta \leq 0$, Eq. (37) is an intial value-problem with the history function $g(t),-\tau \leq t \leq 0$, i.e. $h_{t}(\theta, 0)=g(\theta)$. Then the method of characteristics leads to

$$
h_{t}(\theta, t)=-\int_{0}^{t} \Phi(t+\theta-s) \Psi(0) I(s) d s+H(\theta+t), t+\theta<0,
$$

with $H(\theta)=g(\theta)$. We point out that this solutins holds for the time interval $t \in[0 ;-\theta]$ only.

- for $t+\theta>0$, Eq. (37) is a boundary value-problem at $\theta=0$ and we find by the method of characteristics

$$
h_{t}(\theta, t)=-\int_{0}^{-\theta} \Phi(-s) \Psi(0) I(t+\theta+s) d s+H(\theta+t) .
$$

Indeed, writing Eq.(35) for $\theta=0$ yields

$$
\frac{\partial h_{t}(0, t)}{\partial t}=L\left[h_{t}(t)\right]+(1-\Phi(0) \Psi(0)) I(t) .
$$

According to Eq.(39), we may write $h_{t}(\theta, t)=r(\theta, t)+H(t+\theta)$, where $r(\theta, t)=-\int_{0}^{-\theta} \Phi(-s) \Psi(0) I(t+\theta+s) d s$. Then inserting this expression into (40) leads to

$$
\frac{\partial H(t)}{\partial t}=L[H(t)]+L[r(t)]+(1-\Phi(0) \Psi(0)) I(t)
$$

Recall that $L[H(t)]$ is a functional of $H(\theta+t)$ and thus $L[H(t)]$ may depend on $H(t)$ and $H(t-\tau)$. Similarly $L[r(t)]$ is a functional of $r(\theta, t)$. Given that the functional $r(\theta, t)$ is known and the linear term contains retarded terms, Eq.(41) is a non-autonomous linear delay-differential equation in $H$ which can be solved analytically $[18,29]$.

The approach illustrates the hypothesis that the non-autonomous case can be analyzed, in lowest order approximation, by a fast and small time-dependent correction on the center manifold. Higher degrees of accuracy than the second 
order could be achieved by proceeding to higher order terms in both modes and time dependent components of $h(\theta, u, \varepsilon, t)$ in Eq.(36). The time-mode separable form, i.e. the separation of the mode-dependent part $h_{a}$ and the time-dependent part $h_{t}$, combined with the time-scale separation asumption allows to compute higher order terms in the modes expansion, while keeping the time-dependent component to second order. The subsequent section examines the hypotheses made and the corresponding results by applying the method to a specific example.

\section{The asymmetrical transcritical bifurcation}

To validate our approach, let us apply the procedure discussed in the previous sections to a non-autonomous delayed differential equation with quadratic nonlinearity near a codimension 1 instability. The system we consider reads

$$
\frac{d x(t)}{d t}=-x(t)-R_{1} x(t-\tau)-R_{2} x^{2}(t-\tau)+I(t),
$$

with the augmented system

$$
\begin{aligned}
\frac{d x(t)}{d t} & =-x(t)+x(t-\tau)-\varepsilon x(t-\tau)-R_{2} x^{2}(t-\tau)+I(t) \\
\frac{d \varepsilon}{d t} & =0,
\end{aligned}
$$

where we introduced the control parameter $\varepsilon \equiv R_{1}+1$. Applying the steps described in the previous section we obtain the reduced dynamics [30]

$$
\begin{aligned}
\frac{d u(t)}{d t} & =\frac{1}{1+\tau} F\left[u(t)+s_{t}\right]+\frac{1}{1+\tau} I(t) \\
\frac{d \varepsilon(t)}{d t} & =0 \\
\frac{d}{d t} s_{t}(\theta) & =\mathcal{A}\left(s_{t}\right)+\left(X_{o}-\frac{1}{1+\tau}\right)\left(F\left[u(t)+s_{t}\right]+I(t)\right) .
\end{aligned}
$$

Now applying the center manifold theorem implies that the functional $h$ depends on time explicitely and with the separation ansatz $36 h_{t}$ obeys

$$
\frac{\partial h_{t}}{\partial t}=\frac{\partial h_{t}}{\partial \theta}-\frac{1}{1+\tau} I(t) .
$$

Then Eqs.(38), (39) lead to the solutions

$$
\begin{aligned}
& h_{t}(\theta, t)=-\frac{1}{1+\tau} \int_{0}^{t} I(s) d s+H(\theta+t) \quad, \quad t \leq-\theta \\
& h_{t}(\theta, t)=-\frac{1}{1+\tau} \int_{0}^{-\theta} I(t+\theta+s) d s+H(\theta+t) \quad, \quad t>-\theta,
\end{aligned}
$$


with the initial condition $H(\theta)=g(\theta)$. Substituting these results into Eq.(41) yields the evolution equation of $H(t), t \geq 0$ for $\theta=0$

$$
\frac{d H}{d t}=-H(t)+H(t-\tau)-\frac{1}{1+\tau} \int_{0}^{\tau} I(t-\tau+s) d s+\frac{\tau}{1+\tau} I(t) .
$$

We point out, that the last two terms may be viewed as an external driving and the linear terms are the same as the linear terms in the original system. By virtue of the spectrum of the linear operator the linear system in $H(t)$ in (47) is marginally stable close to the stability threshold (the maximum Lyapunov exponent is close to zero). The other Lyapunov exponents have negative real parts and their contribution vanish for large times.

To verify these results, let us consider the periodic driving $I(t)=I_{o} \sin \left(w_{o} t\right)$, whose amplitude $I_{o}$ is small compared to the amplitude $u(t)$ and whose oscillation period is short compared to the slow evolution of $u(t)$. The autonomous components of the center manifold were found previously. The time-dependent correction is given by Eq.(46)

$$
\begin{aligned}
& h_{t}(\theta, t)=H(t+\theta)+\frac{I_{o}}{w_{o}(1+\tau)}\left(\cos \left(w_{o} t\right)-1\right) \quad, \quad t \leq-\theta \\
& h_{t}(\theta, t)=H(t+\theta)+\frac{I_{o}}{w_{o}(1+\tau)}\left(\cos \left(w_{o} t\right)-\cos \left(w_{o}(t+\theta)\right)\right) \quad, \quad t>\theta .
\end{aligned}
$$

Assuming the initial function $g(t)=0,-\tau \leq t \leq 0$, and for large times $t \rightarrow \infty$, the solution of (47) reads

$$
\begin{aligned}
H(t)= & -\frac{I_{o} \tau}{(1+\tau)^{2} w_{o}}\left(\cos \left(w_{o} t-1\right)\right) \\
& +\frac{\tau I_{o}}{w_{o}^{2}(1+\tau)^{2}}\left(\sin \left(w_{o} t\right)-\sin \left(w_{o}(t-\tau)\right)-\sin \left(w_{o} \tau\right)\right) \\
& +R_{1}\left(I_{o}, w_{o}\right) \sin \left(w_{o} t\right)+R_{2}\left(I_{o}, w_{o}\right) \cos \left(w_{o} t\right) .
\end{aligned}
$$

with constants $R_{1}\left(I_{o}, w_{o}\right)$ and $R_{2}\left(I_{o}, w_{o}\right)$ depending on the stimulus frequency $w_{o}$, the input strength $I_{o}$ and the delay $\tau$.

Taking into account the time-dependence of the center manifold gives access to more than just the good reconstruction of the systems dynamics. In general, nonautonomous components play a major role in the stability of dynamical systems, especially in the vicinity of dynamic instabilities, i.e. in the presence of different time scales $[17,31]$. Therefore, the study of time-corrected center manifolds yield details about input-induced bifurcations. Let us investigate the interaction of the input $I(t)$ with the transcritical bifurcation studied.

Based on the calculations in the previous section, we may write the order parameter equation as

$$
\frac{d u(t)}{d t}=\frac{1}{1+\tau}\left(-\varepsilon(u+h(-\tau, u, t))-R_{2}(u+h(-\tau, u, t))^{2}+I(t)\right),
$$


where we omitted the trivial dynamics of the control parameter $\varepsilon$. Over a finite time interval, we might time-average both sides of Eq.(50) to obtain

$$
\begin{aligned}
\left\langle\frac{d u(t)}{d t}\right\rangle= & \frac{1}{1+\tau}(\langle I(t)\rangle-\varepsilon\langle u\rangle-\varepsilon\langle h(-\tau, u, t))\rangle \\
& \left.\left.-R_{2}\left\langle u^{2}\right\rangle-2 R_{2}\langle u h(-\tau, u, t))\right\rangle-R_{2}\left\langle h(-\tau, u, t)^{2}\right\rangle\right),
\end{aligned}
$$

where the time averaging operator $\langle\cdot\rangle$ is defined over an interval $T$ by

$$
\langle\cdot\rangle=\frac{1}{T} \int_{t}^{t+T}(\cdot) d t .
$$

Following the time scale separation issued by the center manifold theorem and the OPE, the input $I(t)$ is considered fast, compared to the unstable mode $u(t)$. Hence, for $T$ sufficiently small, $u(t)$ is approximately constant, and we may consequently write $\langle u(t)\rangle \approx u(t)$. Thus, if the input is chosen such that $\langle I(t)\rangle=0$, Eq.(51) may be written as

$$
\begin{aligned}
\left\langle\frac{d u(t)}{d t}\right\rangle= & -\frac{1}{1+\tau} R_{2}\left(u^{2}+\left(\frac{\varepsilon}{R_{2}}+2\langle h(-\tau, u, t)\rangle\right) u\right. \\
& \left.+\left(\frac{\varepsilon}{R_{2}}\langle h(-\tau, u, t)\rangle+\left\langle h(-\tau, u, t)^{2}\right\rangle\right)\right) .
\end{aligned}
$$

Because of the separation of the time scales, the fixed points of the averaged equation correspond to the fixed points of Eq.(50). Inserting the stable manifold $h(-\tau, u, t)$ with the terms in Eqs. (48) and (49) found in section 3, we gain the stationary states by setting $\left\langle\frac{d u(t)}{d t}\right\rangle=0$. In addition the focus to the solutions close to the origin, leads to the stationary states

$u^{o}=\frac{1}{2 A_{0}+2 A_{1}\left\langle h_{t}\right\rangle}\left(-\left(B_{0}+B_{1}\left\langle h_{t}\right\rangle\right) \pm \sqrt{\left(B_{0}+B_{1}\left\langle h_{t}\right\rangle\right)^{2}-4\left(A_{0}+A_{1}\left\langle h_{t}\right\rangle\right)\left\langle h_{t}^{2},\right\rangle},\right)$

with functions $A_{0}=A_{0}(\varepsilon), A_{1}=A_{1}(\varepsilon), B_{0}=B_{0}(\varepsilon), B_{1}=B_{1}(\varepsilon)$ and $h_{t}=$ $h_{t}(-\tau, t)$. Since the external input can be viewed as a a linear superposition of oscillations according to Fourier theory and hence $h_{t}$ exhibits oscillations with the same frequencies, it is reasonable to assume that $\left\langle h_{t}\right\rangle \approx 0$. This assumption implies that the time-average window $T$ is smaller than the period of the slowest Fourier component in the input. Then we find

$$
u^{o}=\frac{1}{2 A_{0}}\left(-B_{0} \pm \sqrt{B_{0}^{2}-4 A_{0}\left\langle h_{t}^{2} \cdot\right\rangle}\right)
$$

with $\left\langle h_{t}^{2}\right\rangle \geq 0$. If $\left\langle h_{t}^{2}\right\rangle=0$, then $I(t)=0$ and $u^{o}=0,-B_{0} / A_{0} \approx-\varepsilon \times$ const and the origin $u^{o}$ is the only stationary solution for $\varepsilon=0$. In contrast, if $\left\langle h_{t}^{2}\right\rangle>0$ for $I(t) \neq 0$ and the origin is not a stationary solution of the dynamics. New equilibria are moved to $\varepsilon_{m i n, 1}>0, \varepsilon_{m i n, 2}<0$. These solutions are roots of the 
polynomial $B_{0}^{2}(\varepsilon)-4 A_{0}(\varepsilon)\left\langle h_{t}^{2}\right\rangle=0$. These results demonstrate that the external input destroys stationary states, that existed without external input, and breaks the symmetry of the transcritical bifurcation: the external input changes the stability of the system.

To verify these analytical results, we choose the averaging interval as the period of one input cycle i.e. $T=2 \pi / w_{o}$ so that $\langle I(t)\rangle=0$. Considering the full terms in Eq. (52) and by setting $\left\langle\frac{d u(t)}{d t}\right\rangle=0$, we may find the stationary states numerically, see Fig. 1. We immediately see that whenever $\left\langle h_{t}^{2}\right\rangle>0$, the symmetry of transcritical bifurcation is broken, and we obtain a imperfect bifurcation scenario. This symmetry breaking replaces the intersecting branches by two disjoint saddle-node curves. In order for the order parameter equation to capture this particular bifurcation diagram, the precision of the center manifold $h(\theta, t, u)$ is very important. The time-corrected center manifold brings a considerable amount of accuracy to the OPE, not only by adjusting the phase but also the amplitude of the system's response. Fig. 1 shows how the OPE with time-dependent center manifold reproduces the bifurcation diagram of the original DDE with an improved accuracy compared to the same problem but without any time-dependency on the center manifold. 
a)

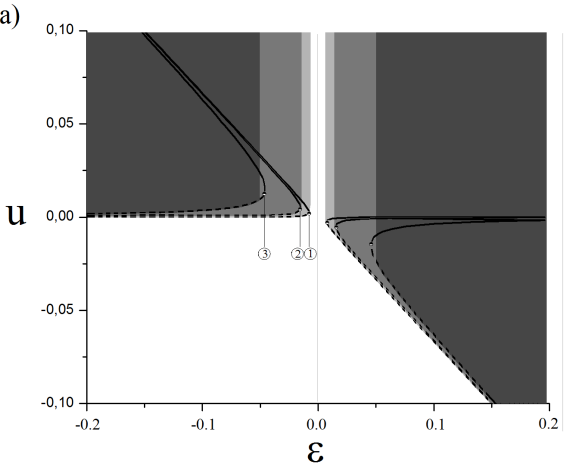

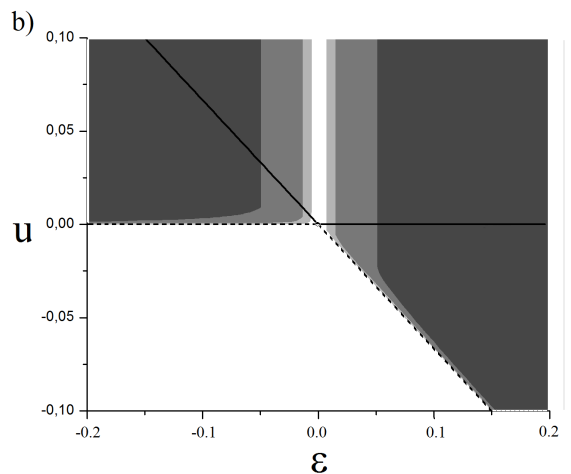

Fig. 1. Bifurcation diagram of the averaged order parameter equation. (a) Comparison of the basins of atttaction of the original DDE for different $I_{o}$ with those predicted applying the proposed time-dependent center manifold reduction. The fixed point curves (solid and dashed lines) of the averaged OPE in Eq.(53) delimits the basins of attraction of the original system (42) shown in tones of shaded gray for different input amplitudes. The input amplitudes have been set to $I_{o}=0.05$ (stationary solutions $\mathbf{1}$ and basin of attraction in light gray), $I_{o}=0.1$ (stationary solutions $\mathbf{2}$ and basin of attraction in gray) and $I_{o}=0.3$ (stationary solutions $\mathbf{2}$ and basin of attraction in dark gray). As the input amplitude increases i.e. $I_{o}>0$, the basin of attraction of the stable fixed points splits and exhibit a band of unstable initial conditions, indicating that the input induces an imperfect transcritical bifurcation. In this case, the stable and unstable branches do not meet at $\varepsilon=0$ as expected and are replaced by two saddle nodes bifurcations. (b) Plot of the fixed point curves predicted by the averaging of the original system (42) where a standard transcritical case is predicted. This result does not correspond to the dynamics of the original system. In contrast, the fixed point curves (53) of the averaged order parameter equation using time-dependent center manifold show saddle node bifurcations for different input amplitudes, delimiting the basins of attraction of the original system accurately for $I_{o}=0.05$ and $I_{o}=0.1$. For $I_{o}=0.3$, the input amplitudes becomes large compared to the unstable mode amplitude. Additional parameters are $w_{o}=15, \tau=2.0, R_{2}=1.5$ 


\section{Concluding Remarks}

In this essay, we showed that the dynamics of a non-autonomous delayed feedback system could be captured by center manifold reduction. This is made possible by allowing an explicit time dependence of the manifold, taking the form of an additive time-dependent correction to the non-driven problem. We illustrated the approach by considering a scalar delay differential equation with quadratic non-linearity, driven by an additive time-periodic term, in the vicinity of a transcritical bifurcations. Numerical experiments are in good agreements with the analytical results. Higher degrees of accuracy could be reached by considering higher order terms in both time and mode dependent components of the center manifold.

It is still unclear how the initial conditions of the original DDE are mapped to those of the OPE. There appears to be discrepancy at $t=0$ between the reconstructed and original flow of the system considered in this example, that we corrected manually to match the initial conditions of both the OPE and DDE. This deviations seems to be due to the yet unknown map from the interval $[-\tau, 0]$ to the initial value problem $x(0)=x_{o}$, induced by the projection onto stable and unstable subspaces. This discrepancy usually decays numerically with the transients. A tentative solution to this problem would require a consideration of the individual stable modes initial value problems, an information that appears to be lost with the application of the center manifold theorem and following approximations.

The time-dependent correction considered here is appropriate for additive driving only, as no time-mode mixing is present at lowest order. We might consequently expect that mutiplicative time-dependent forcing would require a different working ansatz, which would not allow a separation between autonomous and non-autonomous problems, as the example detailed here shows. This case would invariably lead to more involving calculations. 


\section{References}

1. A. Longtin and J. G. Milton. Modelling autonomous oscillations in the human pupil light reflex using nonlinear delay-differential equations. Bull. Math. Biol., 51:605-624, 1989.

2. L. Glass and M.C. Mackey. From Clocks to Chaos: The Rhythms of Life. Princeton University Press, Princeton, 1988.

3. J. Boulet, R. Balasubramaniam, A. Daffertshofer, and A. Longtin. Stochastic two delay-differential model of delayed visual feedback effects on postural dynamics. Phil. Trans. Royal Soc. A, 368(1911):423-438, 2010.

4. A. Hutt, M. Bestehorn, and T. Wennekers. Pattern formation in intracortical neuronal fields. Network: Comput. Neural Syst., 14:351-368, 2003.

5. F. M. Atay and A. Hutt. Neural fields with distributed transmission speeds and constant feedback delays. SIAM J. Appl. Dyn. Syst., 5(4):670-698, 2006.

6. S. Coombes and M.R. Owen. Bumps, breathers, and waves in a neural network with spike frequency adaptation. Phys. Rev. Lett., 94:148102, 2005.

7. I. Franovic, K. Todorovic, N. Vasovic, and N. Buric. Spontaneous formation of synchronization clusters in homogenous neuronal ensembles induced by noise and interaction delays. Phys. Rev. Lett., 108:094101, 2012.

8. S.-A. Campbell. Delay Differential Equations: Recent Advances and New Directions, chapter Calculating Center Manifolds for Delay Differential Equations Using Maple. Spinger-Verlag, New York, 2008.

9. B. Redmond, V.G. LeBlanc, and A . Longtin. Bifurcation analysis of a class of firstorder nonlinear delay-differential equations with reflectional symmetry. Physica $D$, $166: 131-146,2002$.

10. W. Wischert, A. Wunderlin, and A. Pelster. Delay-induced instabilities in nonlinear feedback systems. Physical Review E, 49(1), 1994.

11. M. Schanz and A. Pelster. Synergetic system analysis for the delay-induced hopf bifurcation in the wright equation. SIAM J. Applied Dynamical Systems, 2(3):277296, 2003.

12. H. Haken. Synergetics. Springer, Berlin, 2004

13. G. Schoener and H. Haken. The slaving principle for Stratonovich stochastic differential equations. Z. Phys. B, 63:493-504, 1986.

14. C. Chicone and Y. Latushkin. Center manifolds for infinite dimensional nonautonomous differential equations. Journal of differential equations, 141:356-399, 1997.

15. P. Boxler. A stochastic version of center manifold theory. Probab. Theory Relat. Fields, 83:509, 1989.

16. C. $\mathrm{Xu}$ and A.J. Roberts. On the low-dimensional modelling of Stratonovich stochastic differential equations. Physica A, 225:62-80, 1996.

17. A. Hutt, A. Longtin, and L. Schimansky-Geier. Additive global noise delays turing bifurcations. Phys. Rev. Lett., 98:230601, 2007.

18. J.K. Hale and S.M.V. Lunel. Introduction to functional differential equations. Springer, Berlin, 1993.

19. R. Quesmi, M. Ait Babram, and M.L. Hbid. A maple program for computing a terms of a center manifold, and element of bifurcations for a class of retarded functional differential equations with hopf singularity. Applied Mathematics and Computation, 175:932-968, 2006.

20. S.-A. Campbell and J. Belair. Analytical and symbolically-assisted investigations of hopf bifurcations in delay-differential equations. Canadian Applied Mathematics Quarterly, 3:137-154, 1995. 
21. T. Faria and L. Magalhaes. Normal forms for retarded functional differential equations with parameters and applications to hopf bifurcation. Journal of Differential Equations, 122:281, 1995.

22. F.M. Asl and A.G. Ulsoy. Analysis of a system of linear delay differential equations. Journal of Dynamic Systems, Measurement, and Control, 125, 2003.

23. J.C. Li and C.H. Hansen. Forced phase-locked response of a nonlinear system with time delay after hopf bifurcation. Chaos, Solitons and Fractals, 25:461-473, 2005.

24. J. Xu and K.W. Chung. Effects of time delayed position feedback on a van der pol2̆013duffing oscillator. Physica D, 180:17-39, 2003.

25. T. D. Frank and P. J. Beek. Stationary solutions of linear stochastic delay differential equations: Applications to biological systems. Phys. Rev. E, 64:021917, 2001.

26. S. Guillouzic, I. L'Heureux, and A. Longtin. Small delay approximation of stochastic delay differential equation. Phys. Rev. E, 59(4):3970, 1999.

27. N. Yeganefar, P. Pepe, and M. Dambrine. Input-to-state stability of time-delay systems : A link with exponential stability. IEEE Transactions On Automatic Control, 53:1526-1531, 2008.

28. S.M. Cox and A.J. Roberts. Center manifolds of forced dynamical systems. J. Austral. Math. Soc. Ser. B, 32:401-436, 1991.

29. A. Amann, E. Schoell, and W. Just. Some basic remarks on eigenmode expansions of time-delay dynamics. Physica A, 373:191-202, 2007.

30. J. Lefebvre, A. Hutt, V. G. LeBlanc, and A. Longtin. Reduced dynamics for delayed systems with harmonic or stochastic forcing. Chaos, 22:043121, 2012.

31. A. Hutt, A. Longtin, and L. Schimansky-Geier. Additive noise-induced turing transitions in spatial systems with application to neural fields and the swift-hohenberg equation. Physica D, 237:755-773, 2008.

32. H. Haken. Advanced Synergetics. Springer, Berlin, 1983.

33. A. Hutt, J. Lefebvre, and A. Longtin. Delay stabilizes stochastic systems near an non-oscillatory instability. Europhys. Lett., 98:20004, 2012.

34. R. Bellmann and K.L. Cooke. Differential-Difference Equations. Academic Press, New York, 1963.

35. J. Carr. Applications of Center Manifold Theory. Applied Mathematical Sciences 35. Springer verlag, New York, 1981.

36. O. Arino, M.L. Hbid, and E. Ait Dads. Delay Differential Equations and Applications. NATO science series. Springer Verlag, 1999.

37. J. Guckenheimer and P. Holmes. Nonlinear Oscillations, Dynamical Systems and Bifurcation of Vector Fields. Applied Mathematical Sciences 42. Spinger Verlag, 1983.

38. R. Quesmi, M. Ait Babram, and M.L. Hbid. Center manifolds and normal forms for a class of retarded functional differential equations with parameter associated with fold-hopf singularity. Applied Mathematics and Computation, 181:220-246, 2006 .

39. G. Orosz. Hopf bifurcation calculations in delayed systems. Periodica Polytechnica Ser. mech. Eng., 48(2):198-200, 2004.

40. X.-P. Yan and W.-T. Li. Hopf bifurcation and global periodic solutions in a delayed predator-prey system. Applied Mathematics and Computation, 177:427-445, 2006.

41. S.A. Campbell et al. Limit cycles, tori and complex dynamics in a second-order differential equation with delayed negative feedback. J. Dyn. Diff. Eq., textbf7, 213 (1995).

42. J. Bechhoefer. Feedback for physicists: A tutorial essay on control. Reviews of Modern Physics, 77, 2005. 